\title{
Investigation of Spatially Unresolved Magnetic Field Outside Sunspots Using Hinode/SOT Observations
}

\author{
Olga Botygina $^{1}$, Mykola Gordovskyy $^{2}$ and Vsevolod Lozitsky ${ }^{1}$ \\ ${ }^{1}$ Astronomical Observatory of Taras Shevchenko National University of Kyiv Observatorna \\ str., 3, Kyiv 04053, Ukraine \\ email: olga.botygina@gmail.com \\ ${ }^{2}$ Jodrell Bank Centre for Astrophysics, University of Manchester, Alan Turing building, \\ Manchester M13 9PL, United Kingdom
}

\begin{abstract}
The structure of photospheric magnetic fields outside sunspots is investigated in three active regions using Hinode/Solar Optical Telescope(SOT) observations. We analyze Zeeman effect in FeI 6301.5 and FeI 6302.5 lines and determine the observed magnetic field value $B_{\text {eff }}$ for each of them. We find that the line ratio $B_{\text {eff }}(6301) / B_{\text {eff }}(6302)$ is close to 1.3 in the range $B_{\text {eff }}<0.2 k G$, and close to 1.0 for $0.8 k G<B_{\text {eff }}<1.2 k G$. We find that the observed magnetic field is formed by flux tubes with the magnetic field strengths $1.3-2.3 k G$ even in places with weak observed magnetic field fluxes. We also estimate the diameters of smallest magnetic flux tubes to be $15-20 \mathrm{~km}$.
\end{abstract}

Keywords. Sun: magnetic fields, photosphere

\section{Introduction}

Magnetic field in the solar atmosphere is very inhomogeneous on scales, which, at present, cannot be spatially resolved $(<0.2$ arcsec, see review by Stenflo 2013 and references therein). There is observational evidence that the magnetic fields of the quiet photosphere have three components: collapsed, uncollapsed and hidden magnetic flux (Stenflo 2011). The first two components exist in form of strong (collapsed) and weak (uncollapsed) fluxes and can be investigated using the Zeeman effect. The third component exist in form of turbulent fields of $10-100 G$ range and can be observed using the Hanle effect. The magnetic field in the strong component, most likely, exists, in the form of small-scale flux tubes with $k G$ fields. The physical parameters of these flux tubes, such as the real field strength, field distribution, their diameters, plasma characteristics are still unknown.

In this paper, we use the methodology suggested by Cerdena et al. 2003, Botygina et al. 2016 and Lozitsky et al. 2016 and estimate the real field strengths and diameters of the small-scale flux tubes using the Hinode spectrapolarimetric data.

\section{Data and analysis}

We investigate unresolved magnetic field structure by comparing Zeeman splitting of $I+V$ and $I-V$ Stokes profiles in two spectral lines: Fe I 6301.5 and $6302.5 \AA$. These lines are formed at close heights in the solar photosphere but have different magnetic sensitivities (or Lande factors, $g$ ), 1.67 and 2.5, respectively. 

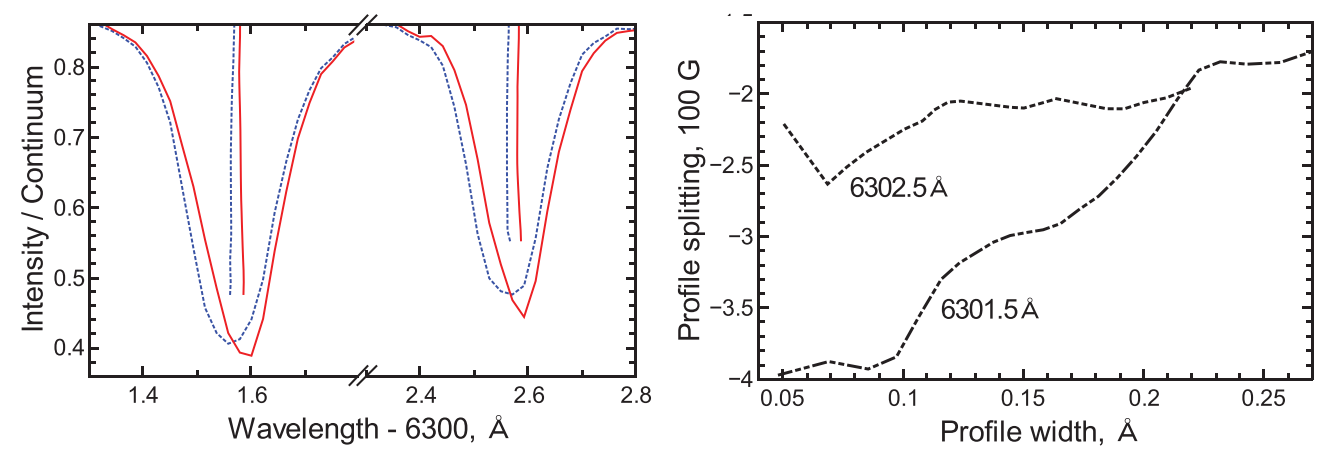

Figure 1. Left panel: $I+V$ and $I-V$ Stokes profiles of 6301.5 and 6302.5 lines, along with their bisectors. Red solid line and blue dashed lines correspond to the $I+V$ and $I-V$ components, respectively. Right panel: Bisector splitting as function of the profile widths for 6301.5 line (dot-dot-dashed line) and 6302.5 line (dashed line).

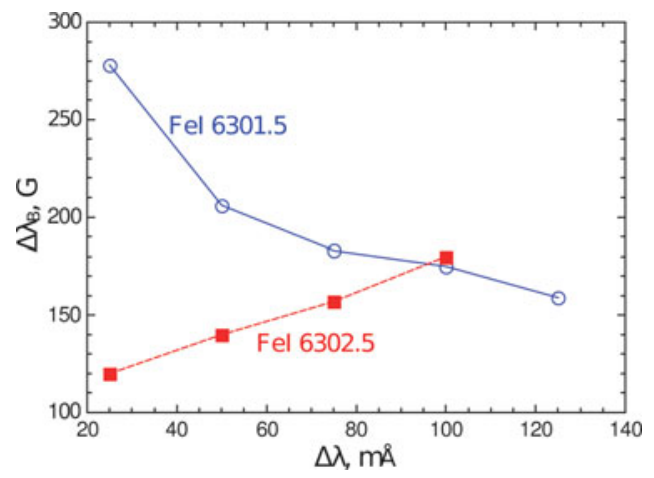

Figure 2. Splitting of the bisectors $\Delta \lambda_{\mathrm{B}}$ in lines FeI 6301.5 (solid blue line) and $6302.5 \AA$ (dashed red line) as functions of the distance from the line centers $\Delta \lambda$ of these lines.

In uniform field $B_{\mathrm{ft}}$ we expect:

- Zeeman splitting in these two lines $\left(B_{\text {eff }}\right)$ to be equal to the real field value, $B_{\text {eff }}=$ $B_{\mathrm{ft}}$, where $B_{\text {eff }}$ represents the magnetic flux going through each pixel, i. e. the measured effective magnetic field;

- cores and wing will show same Zeeman splitting.

However, when the field is non-uniform and contains at least two different spatially unresolved components with strengths $B_{0}$ and $B_{1}$ we expect:

- the observed field values to be $B_{0}<B_{\text {eff }}<B_{1}$;

- the observed field values to be different in Fe I 6301.5 and 6302.5 lines, because of the saturation effect (Botygina et al. 2016), typically $B_{\text {eff }}(6301.5) / B_{\text {eff }}(6301.5)>1$ outside sunspots;

- the cores and wings of these lines to show different splitting (see Fig. 2).

Cerdena et al. 2003 studied the structure of the magnetic field in the center of the disc outside the photospheric grid. It has been found that even with high spatial resolution $(<$ 1 arc second) magnetic field values, obtained by the splitting 6301.5 and 6302.5 lines, are different. It means that magnetic field is inhomogeneous on the scale smaller than $500 \mathrm{~km}$. In addition, the authors calculated the relationship between $B_{\text {eff }}(6301.5) / B_{\text {eff }}(6302.5)$ ratio and the real value in the field of small-scale components for different models of the atmosphere. If magnetic field is about $500 \mathrm{G}$, the $B_{\text {eff }}(6301.5) / B_{\text {eff }}(6302.5) \approx 1$. When 


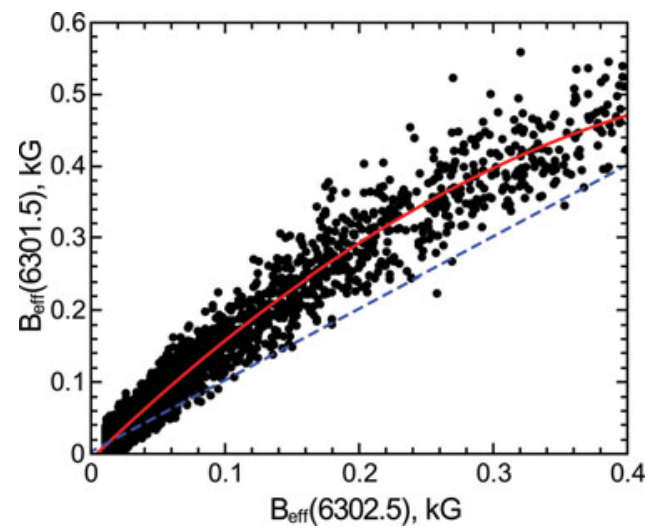

Figure 3. Magnetic fields measured using FeI 6301.5 line versus magnetic fields measured using 6302.5 line. The dashed line corresponds to $B_{\text {eff }}(6301.5) / B_{\text {eff }}(6302.5)=1$. The solid line corresponds to the second order polynomial fit of the data. Colour figures are available in the online version.

value of magnetic field is about $1 k G$ the ratio $B_{\text {eff }}(6301.5) / B_{\text {eff }}(6302.5) \approx 0.8-1.1$, and when the magnetic field is $1.5 \mathrm{kG}$, this ratio increases to $1.2-1.6$.

The study by Cerdena et al. 2003 is based on the assumption that the magnetic field in the small-scale flux tubes is constant (rectangular profile). However, Lozitsky et al. 1989 showed that the field in the flux tubes should be similar to sunspots and pores, with the maximum field strength at the flux tube axis being approximately 1.5 times higher than the average field.

Applying the calibration from Cerdena et al. 2003 to the observed $B_{\text {eff }}(6301) / B_{\text {eff }}(6302)$ ratio, we find that the average field in the flux tubes is about $1.5-1.6 \mathrm{kG}$. Taking into account the non-uniformity of the field within the flux tubes, the maximum field strength should be about $2.0-2.4 k G$.

Now, let us estimate flux tube diameters assuming the two-component magnetic field structure. The observed field $B_{\text {eff }}$ should depend on the background field $B_{\text {backgr }}$ and flux tube field $B_{\mathrm{ft}}$ as

$$
B_{\mathrm{eff}}=(1-f) B_{\mathrm{backgr}}+f k K Z B_{\mathrm{ft}}
$$

where $f$ is the filling factor, $K$ is the parameter accounting for the brightness difference inside and outside flux tubes, $Z$ is the parameter accounting for the saturation effect in different lines. (The factor $Z$ is different for different spectral lines and that is why the resulting $B_{\text {eff }}$ is different for 6301 and 6302 lines.) Here $k=1$ corresponds to a rectangular profile and $k<1$ corresponds to non-rectangular profiles, i.e. similar to those observed in sunspots. We assume that some pixels do not contain any flux tubes at all (i.e. ambient field only), some of them contain only one flux tube and so on. The pixels without flux tubes should yield $B_{\text {eff }}(6301.5)=B_{\text {eff }}(6302.5)$, while pixels with at least one flux tube should yield $B_{\text {eff }}(6301.5)>B_{\text {eff }}(6302.5)$. This can be seen in Fig. 3: $B_{\text {eff }}(6301.5) \approx B_{\text {eff }}(6302.5)$ below $15-20 G$, while in the region $50-100 G$ the ratio $B_{\text {eff }}(6301.5) / B_{\text {eff }}(6301.5)$ is about 1.3 .

Because the filling factor is expected to be small (Botygina et al. 2016), one can approximate $(1-f) B_{\text {backgr }} \approx B_{\text {backgr }}$. Hence, formula $(2.1)$ can be rewritten as

$$
B_{\text {eff }} / f=B_{\text {backgr }} / f+k K Z B_{\mathrm{ft}}
$$

In the pixel with only one flux tube, the filling factor is $f=\frac{p i}{4} \frac{d^{2}}{S_{0}}$, where $d$ is the flux tube diameter, and $S_{0}$ is the area of the photosphere resolved by the telescope. Substituting 
this into formula (2.2), the formula for flux tube diameters can be written as:

$$
d \approx 2 \sqrt{\frac{B_{\mathrm{eff}, \min } S_{0}}{\pi\left(B_{\mathrm{backgr}} / f+k K Z B_{\mathrm{ft}}\right)}},
$$

where $f$ is filling factor for flux tubes and $k$ is a factor accounting for the radial distribution of magnetic field $B(x)$ in flux tubes, $Z$ is the factor accounting for Zeeman splitting; $B_{\text {eff,min }}$ is the effective magnetic field, which correspond to the presence of only one flux tube inside aperture, $S_{0}$ is the equivalent area of the input aperture for direct observations. The strength of background field, $B_{\text {backgr }}$, and filling factor of flux tubes, $f$, are connected via simple formula: $B_{\text {backgr }} / f \approx 1 k G$. The ratio $B_{\text {backgr }} / f \approx 1 k G$ should be approximately $1000 \mathrm{G}$ (Lozitsky et al. 1989). Taking into account that direct resolution for Hinode/SOT is $230 \mathrm{~km}$, we have $S_{0}=4.15 \times 10^{4} \mathrm{~km}^{2}$, assuming a circular entrance aperture of the instrument. As it was explained above, $B_{\mathrm{eff}, \min }=10-20 \mathrm{G}$.

Parameters $K \approx 2, k \approx 2 / 3$ and $Z \approx 0.5-0.75$. Therefore, with $B_{\mathrm{ft}}=10-20 G$, the product $k K Z B_{\mathrm{ft}} \approx 1.5 k G$. (see Lozitsky et al. 1989). Substituting these values into the formula $(2.3)$, we get $d=14.5 \mathrm{~km}$ for $B_{\text {eff }, \min }=10 \mathrm{G}$ and $d=20.6 \mathrm{~km}$ for $B_{\text {eff }, \text { min }}=20 G$. Formula $(2.1)$ is based on a certain (non-constant) distribution of magnetic field within flux tubes, which is described by parameter $k$. Based on this model, for the active region NOAA 2339 we find that the sizes of smallest flux tubes are $\approx 15-20 \mathrm{~km}$.

\section{Conclusions}

By applying the approach described in Botygina et al. 2016 and Lozitsky et al. 2016 we find that

- The actual strength of local magnetic fields in flux tubes is about $2.0-2.3 \mathrm{kG}$;

- A weak, nearly uniform field is found in pixels $B_{\text {eff }}<10-20 G$ (Fig. 3).

- The diameter of smallest flux tubes in the considered active region is about $15-$ $20 \mathrm{~km}$.

It should be noted that these estimations are subject to the model assumptions. We believe, the obtained sizes are diameters of smallest flux tubes in the regions. If the sizes of flux tubes are that small, they would not be resolved by the next generation of solar telescopes, such as GREGOR (see Schmidt et al. 2012) and, hence, spectropolarimetric techniques will need to be used for diagnostics. The value $B_{\text {backgr }} / f$ is known to the order of magnitude. However, this value changes the resulting diameter $d$ only by factor $\approx 2\left(d \approx 40 \mathrm{~km}\right.$ when $\left.B_{\text {backgr }} / f=0\right)$.

\section{References}

Botygina, O., Gordovskyy, M., \& Lozitsky, V., 2016, Advances in Astronomy and Space Physics, Volume 6, Issue 1, 20

Cerdena, D., Almeida, J.S. \& Kneer, F., 2003 A $\& A$, 407, 741

Lozitsky, V., Botygina, O., \& Gordovskyy, M., 2016, Astron. Tsirkular No. 1631, 1

Lozitsky, V., \& Tsap, T., 1989, Kinematika i Fizika Neb. Tel, V.5, 1, 50

Schmidt, W., Lühe von der O., Volkmer, R., et al. 2012, Astron. Nachrichten, 333, 9, 796

Solanki, S., \& Stenflo, J., 1984, Astron. Astrophys., 140, 185

Stenflo, J., 2011, A \& A, 529, A42

Stenflo, J., 2013, Astron. Astrophys. Rev., 21, 66 